\title{
Obtención de metano por medio de la combinación de excretas de vacas y conejos
}

\section{Obtaining methane through the combination excreta of cows and rabbits}

\author{
HERNÁNDEZ-GÓMEZ, Víctor Hugo †*, OLVERA-GARCÍA, Omar y OAXACA-BELTRÁN, \\ Marlene
}

Universidad Nacional Autónoma de México, FES Cuautitlán, Estado de México, México

ID $1^{\text {er }}$ Autor: Víctor Hugo, Hernández-Gómez / ORC ID: 0000-0001-9315-5869, Researcher ID Thomson: S-6575-2018, CVU CONACYT ID: 122247

ID $1^{\text {er }}$ Coautor: Omar, Olvera-García / ORC ID: 0000-0001-6386-9772, Researcher ID Thomson: S-6644-2018, CVU CONACYT ID: 706478

ID $2^{\text {do }}$ Coautor: Marlene, Oaxaca-Beltrán / ORC ID: 0000-0001-8363-900X, Researcher ID Thomson: 1-8363-900X, CVU CONACYT ID: 986236

DOI: $10.35429 / J C P E .2019 .21 .6 .19 .24$

Recibido 09 Agosto, 2019; Aceptado 30 Noviembre, 2019

\section{Resumen}

El trabajo muestra la producción de metano que se obtuvo al combinar diferentes sustratos de excretas de vaca y conejo. Se empleó para el estudio, cinco biodigestores del tipo Batch, en los cuales fueron llenados con diferentes proporciones de excretas de vaca, conejo y agua, con la finalidad de cuantificar que combinación arrojaba mayor cantidad de metano. Con la finalidad de conocer la cantidad de materia libre de humedad y la fracción que ha de producir biogás, se determinó la masa seca y masa volátil de las excretas de vaca y conejo. Durante la prueba se tomaron lecturas de $\mathrm{pH}$ con tiras reactivas, presión con manómetro, temperatura con termómetro digital y composición del biogás con un analizador de biogás. Al final se obtuvo que el sustrato conformado por $10 \%$ de excretas de vaca, $40 \%$ de excretas de conejo y $50 \%$ agua, presentó la mayor cantidad de metano de todas las combinaciones.

Biodigestor, Biogás, Metano

\begin{abstract}
The work shows the methane production that was obtained by combining different substrates of cow and rabbit excreta. Five biodigesters of the Batch type were used for the study, in which they were filled with different proportions of cow, rabbit and water excreta, in order to quantify which combination produced the greatest amount of methane. In order to know the amount of moisture free material and the fraction that has to produce biogas, the dry mass and volatile mass of cow and rabbit excreta were determined. During the test, readings of $\mathrm{pH}$, pressure, temperature and composition of the biogas were taken. In the end it was obtained that the substrate formed by $10 \%$ of cow excreta, $40 \%$ of rabbit excreta and $50 \%$ water, presented the highest amount of methane of all the combinations.
\end{abstract}

Biodigester, Biogás, Methane

Citación: HERNÁNDEZ-GÓMEZ, Víctor Hugo, OLVERA-GARCÍA, Omar y OAXACA-BELTRÁN, Marlene. Obtención de metano por medio de la combinación de excretas de vacas y conejos. Revista de Energía Química y Física. 2019. 6-21: 1924

\footnotetext{
* Correspondencia al Autor (Correo electrónico: vichugo@unam.mx)

$\dagger$ Investigador contribuyendo como primer Autor
} 


\section{Introducción}

La Facultad de Estudios Superiores Cuautitlán, donde se imparten las carreras de Médico Veterinario Zootecnista y la de Ingeniero Agrícola, para cubrir las actividades académicas de estos alumnos, cuenta con grandes áreas compuestas por establos, corrales, invernaderos, tierras para cultivo (maíz, alfalfa, sorgo, cebolla, vides y nopal), etc. Los desechos orgánicos producto de los animales, así como de las actividades de cultivo, son depositados a cielo abierto, provocando que, al descomponerse, liberen el biogás a la atmosfera.

El biogás es un gas de alto poder calorífico compuesto principalmente por metano $\left(\mathrm{CH}_{4}\right)$, dióxido de carbono $\left(\mathrm{CO}_{2}\right)$, nitrógeno $\left(\mathrm{N}_{2}\right)$ y ácido sulfúrico $\left(\mathrm{H}_{2} \mathrm{~S}\right)$, así como otros gases en proporciones menores producido por la degradación anaeróbica (en ausencia de oxigeno) de la fracción de materia orgánica presente en los residuos [1].

Con la finalidad de aprovechar dichos residuos, se han realizado proyectos en los cuales se ha detectado el porcentaje de metano que se puede producir con los desechos orgánicos presentados en la tabla 1.

\begin{tabular}{|l|}
\hline Materia Orgánica \\
\hline Poda de pastos \\
\hline Excremento de conejo \\
\hline Excremento de borrego \\
\hline Excremento de vaca \\
\hline Desechos de comida \\
\hline Excremento de caballo \\
\hline Excremento de cabra \\
\hline
\end{tabular}

Tabla 1 Materia orgánica de la FES Cuautitlán Fuente: Elaboración Propia

Se encontró que el excremento de vaca produce la mayor cantidad de biogás, pero el excremento de conejo produce el mayor porcentaje de metano (mayor al 70\%) con poca producción de biogás. Algunas materias orgánicas como la poda de pastos y los desechos de comida se vieron influenciado por un $\mathrm{pH}$ muy acido que inhibió por completo el proceso de fermentación anaeróbica, y en algunos otros la temperatura disminuyo drásticamente ocasionando que la producción de biogás disminuyera.
El objetivo de este proyecto consiste en determinar, que concentración de materia fecal de conejo y vaca, puede generar altos volúmenes de biogás con alto porcentaje de metano, considerando los resultados obtenidos en los proyectos anteriores. Con esto se pretende contribuir a la FESC de dos formas, una llevando metano a los laboratorios y la otra como calentamiento de agua a través de la caldera del gimnasio. Esto da como resultado ahorro en el consumo de energéticos.

\section{Antecedentes}

En 1876, Herter reportó que el acetato encontrado en las aguas residuales, estequiométricamente forma metano y dióxido de carbono en cantidades iguales. Louis Pasteur intentó en 1884 producir biogás a partir de estiércol de caballo recogido en las carreteras de París. Junto con su estudiante Gavon consiguió producir $100 \mathrm{~L}$ de metano a partir de $1 \mathrm{~m}^{3}$ de estiércol fermentado a $35^{\circ} \mathrm{C}$. Pasteur alegó que esta tasa de producción debería ser suficiente para cubrir las necesidades energéticas del alumbrado público de París. La aplicación de la energía de los recursos renovables comenzó a partir de ese momento [2], desde entonces se han realizado diferentes estudios. En la FES Cuautitlán se han realizado estudios para la obtención de materia seca, materia volátil, producción de metano, como sigue:

Hernández y et al [3] determinaron que con los desechos orgánicos se podría cubrir la demanda eléctrica de un rancho ganadero en la comunidad de San Bartolo Cuautlalpan, se determinó el potencial de generación de biogás que se podía obtener y se dio una propuesta para el sistema de generación de energía eléctrica.

Hernández y et al [4] presentan el diseño de dos biodigestores continuos para la FES Cuautitlán, empleando los resultados obtenidos al realizar pruebas experimentales con estiércol de vaca y conejo. Determinan el tiempo de retención hidráulica y el posible volumen total de metano.

Hernandez y et al [5] estudiaron el comportamiento de la generación de metano, empleando los desechos orgánicos de la FES Cuautitlán. Determinaron masa seca, masa volátil, tiempo de retención y porcentaje de metano de 12 sustratos diferentes. 


\section{Estudio experimental}

a) Metodología y procedimiento experimental. Para el estudio experimental se realizó la siguiente metodología:

Se emplearon los biodigestores diseñados dentro del laboratorio de Investigación en Energías Renovables de la FES Cuautitlán, cada uno cuenta con una capacidad de 33 litros y son del tipo Batch. Fueron llenados en una sola ocasión y sólo se sacaron muestras para las tomas de $\mathrm{pH}$ y análisis de biogás.

Se recolectaron muestras de excreta de conejo y vaca, para obtener la respectiva masa seca y masa volátil. Las muestras se depositaron en una mufla durante $48 \mathrm{hr}$. a $105 \mathrm{C}$, con el fin de eliminar la humedad contenida en la excreta. Los resultados obtenidos se presentan en la tabla 2.

\begin{tabular}{|l|r|}
\hline Muestra de excremento & \multicolumn{1}{c}{$\begin{array}{c}\text { Masa deshidratada } \\
\left(\mathrm{M}_{\text {total }}-\mathrm{M}_{\text {recipiente }}\right)(\mathrm{g})\end{array}$} \\
\hline Bobino & 18.6 \\
\hline Conejo & 32.9 \\
\hline
\end{tabular}

Tabla 2 Valores de masa deshidratada Fuente: Elaboración Propia

Las muestras se introdujeron de nuevo a la mufla, esta vez incrementando la temperatura y reduciendo el tiempo de espera, es decir a 550 C durante 6 hr. En la tabla 3 se presentan los resultados obtenidos.

\begin{tabular}{|l|r|}
\hline Muestra de excremento & \multicolumn{2}{|l|}{$\begin{array}{l}\text { Masa incinerada } \\
\left.\text { (Mtotal- } \mathbf{M}_{\text {recipiente }}\right)(\mathbf{g})\end{array}$} \\
\hline Bobino & 3.7 \\
\hline Conejo & 5.3 \\
\hline
\end{tabular}

Tabla 3 Valores de masa incinerada Fuente: Elaboración Propia

Con los valores de la tabla 3 , se determinó la masa seca y masa volátil, los resultados se presentan en la tabla 4.

\begin{tabular}{|l|c|c|}
\hline \multicolumn{1}{|c|}{$\begin{array}{c}\text { Muestra de } \\
\text { excremento }\end{array}$} & $\begin{array}{c}\text { Masa seca } \\
\text { (\%) }\end{array}$ & $\begin{array}{c}\text { Masa volátil } \\
\text { (\%) }\end{array}$ \\
\hline Bobino & 12.79229711 & 80.10752688 \\
\hline Conejo & 34.27083333 & 83.89057751 \\
\hline
\end{tabular}

Tabla 4 Valores de la masa seca y volátil de las diferentes excretas

Fuente: Elaboración Propia
Para el caso del bobino el $12.79 \%$ de estiércol es materia libre de humedad y el $80.10 \%$ de ella debería ser capaz de convertirse en biogás. En cambio, para el conejo el $34.27 \%$ de estiércol es materia libre de humedad y el $83.89 \%$ de ella es materia seca.

Para determinar la cantidad necesaria de sustrato en las mezclas, se utilizó la densidad, lo cual permite calcular de manera sencilla, parámetros como la carga orgánica o la dilución del sustrato, entre otros. Para determinar la densidad del excremento, primero se obtuvo el volumen de la excreta, el cual se presenta en la tabla 5.

\begin{tabular}{|l|c|c|}
\hline \multicolumn{1}{|c}{$\begin{array}{c}\text { Muestra de } \\
\text { excremento }\end{array}$} & $\begin{array}{c}\text { Volumen } \\
\left(\boldsymbol{m}^{\mathbf{3}}\right)\end{array}$ & $\begin{array}{c}\text { Densidad } \\
\left(\frac{\boldsymbol{k g}}{\boldsymbol{m}^{\mathbf{3}}}\right)\end{array}$ \\
\hline Bobino & 0.0001864 & 780.0429185 \\
\hline Conejo & 0.0001675 & 573.1343284 \\
\hline
\end{tabular}

Tabla 5 Volumen y densidad de las diferentes excretas Fuente: Elaboración Propia

El porcentaje óptimo de materia sólida en la mezcla contenida dentro del biodigestor, debe estar entre el $8 \%$ al $12 \%$. Esta proporción se logra mezclando la materia orgánica con agua. La dilución de la materia orgánica es importante para generar un mejor ambiente para las bacterias en el sustrato.

Cuando la masa seca de la materia prima con la que se alimenta un biodigestor es mayor a dicho intervalo, es necesario agregar cierta cantidad de agua con la intención de lograr una concentración óptima. En la tabla 6 se presentan los valores de agua presente en el sustrato.

\begin{tabular}{|l|r|r|}
\hline $\begin{array}{l}\text { Muestra de } \\
\text { excremento }\end{array}$ & \multicolumn{1}{c}{$\begin{array}{c}\text { Masa } \\
\text { fresca } \\
(\mathbf{g})\end{array}$} & $\begin{array}{r}\text { Masa de agua presente } \\
\text { en el sustrato }(\boldsymbol{k g})\end{array}$ \\
\hline Bobino & 145.4 & 0.009599999862 \\
\hline Conejo & 96 & 0.178166666 \\
\hline
\end{tabular}

Tabla 6 Valores de la masa de agua presente en el sustrato Fuente: Elaboración Propia

La tabla 6 indica que por cada $0.1454 \mathrm{~kg}$ de estiércol fresco de vaca se debe agregar $0.0096 \mathrm{~kg}$ de agua y por cada $0.096 \mathrm{~kg}$ de estiércol fresco de conejo se debe agregar 0.1782 $\mathrm{kg}$ de agua. En la práctica resulta más sencillo cuantificar el estiércol y el agua de manera volumétrica que de manera másica, por lo tanto: $1 \mathrm{~L}$ de estiércol de vaca $\rightarrow 0.051 \mathrm{~L}$ de agua. $1 \mathrm{~L}$ de estiércol de conejo $\rightarrow 1.063 \mathrm{~L}$ de agua. 
Con base en lo anterior, las mezclas empleadas para el estudio se presentan en la tabla 7.

\begin{tabular}{|l|r|r|r|}
\hline $\begin{array}{l}\text { Biodige } \\
\text { stor } \mathbf{N}^{\circ}\end{array}$ & \multicolumn{1}{c|}{$\begin{array}{c}\text { Excremento } \\
\text { de vaca (\%) }\end{array}$} & $\begin{array}{r}\text { Excremento } \\
\text { de conejo (\%) }\end{array}$ & $\begin{array}{r}\text { Porcentaje } \\
\text { de agua (\%) }\end{array}$ \\
\hline $\mathbf{1}$ & 71 & 0 & 29 \\
\hline $\mathbf{2}$ & 40 & 10 & 50 \\
\hline $\mathbf{3}$ & 25 & 25 & 50 \\
\hline $\mathbf{4}$ & 10 & 40 & 50 \\
\hline $\mathbf{5}$ & 0 & 50.044 & 49.955 \\
\hline
\end{tabular}

Tabla 7 Propuestas de las diferentes mezclas con diferentes porcentajes

Fuente: Elaboración Propia

Con la finalidad de validar las concentraciones empleadas en los cinco biodigestores, se calculó el porcentaje de masa seca presente en cada uno de los sustratos. Los valores obtenidos para cada mezcla se presentan en la tabla 8.

Se puede observar que las mezclas se encontraron dentro del rango aceptable de masa seca, por lo que se procedió al llenado y sellado de cada biodigestor. La prueba se realizó en 149 días.

\begin{tabular}{|l|r|r|r|r|}
\hline $\begin{array}{c}\# B \mathbf{B i} \\
\mathbf{0}\end{array}$ & $\begin{array}{c}\text { Excremento de } \\
\text { vaca (kg) }\end{array}$ & $\begin{array}{c}\text { Excremento de } \\
\text { conejo }(\mathbf{k g})\end{array}$ & $\begin{array}{c}\text { Agua } \\
(\mathbf{k g})\end{array}$ & $\begin{array}{c}\text { Masa seca } \\
(\mathbf{\%})\end{array}$ \\
\hline $\mathbf{1}$ & 5.4586 & 0 & 3.002 & 8.25 \\
\hline $\mathbf{2}$ & 3.1202 & 0.5731 & 5 & 8.44 \\
\hline $\mathbf{3}$ & 1.9501 & 1.4328 & 5 & 11.2 \\
\hline $\mathbf{4}$ & 0.780 & 2.2925 & 5 & 12.500 \\
\hline $\mathbf{5}$ & 0 & 2.8682 & 4.996 & 12.500 \\
\hline
\end{tabular}

Tabla 8 Porcentaje de masa seca presente en cada sustrato Fuente: Elaboración Propia

b) Instrumentación.

Para la lectura de las variables del proceso se emplearon los siguientes equipos: Para la presión se emplearon dos manómetros, uno de baja presión (menor a 2 psi) y otro hasta 14 psi; $\mathrm{La}$ temperatura fue recabada mediante termopares (instalados en la tapa de cada biodigestor) conectados a termómetros digitales; El $\mathrm{pH}$ se midió mediante tiras reactivas de tornasol; y mediante el analizador de gases marca MADUR, con sensores NDIR y electrolíticos, se realizaron mediciones de metano $\left(\mathrm{CH}_{4}\right)$, dióxido de carbono $\left(\mathrm{CO}_{2}\right)$, ácido sulfhídrico $\left(\mathrm{H}_{2} \mathrm{~S}\right)$ y oxigeno $\left(\mathrm{O}_{2}\right)$ del biogás generado dentro de los biodigestores.

\section{Resultados y discusión}

En la figura 1, se muestran las presiones alcanzadas por los biodigestores durante la prueba.

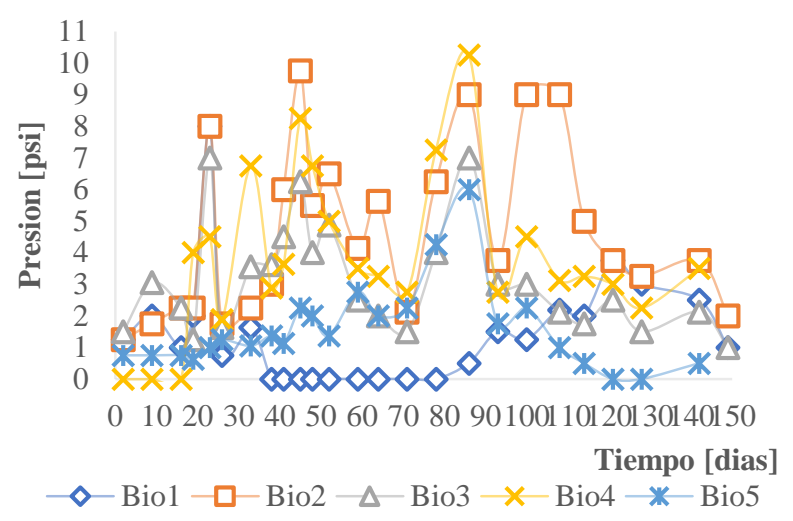

Figura 1 Presión acumulativa alcanzada Fuente: Elaboración Propia

Se observa que el biodigestor que alcanzo una mayor presión fue donde el sustrato estaba compuesto por $10 \%$ de vaca, $40 \%$ de conejo y $50 \%$ de agua, (bio4) teniendo una presión de 10.25 psi, en un tiempo de 86 días, después de dicho día la presión comenzó a decaer, como segundo lugar se tiene que la mezcla con un porcentaje de $0 \%$ de vaca, $50.04 \%$ de conejo y $49.95 \%$ agua, (bio2) alcanzo una presión de 9.75 psi, en un tiempo de 45 días, después empezó a decaer. En la figura 2 se presenta la producción de biogás obtenida por los biodigestores.

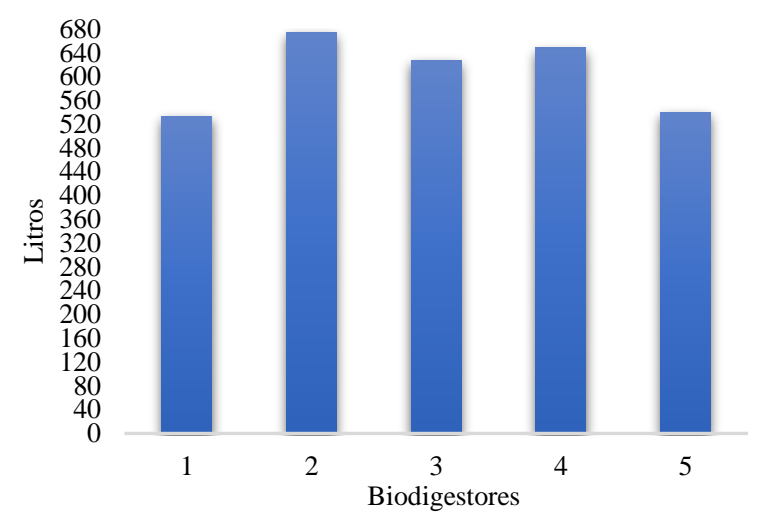

Figura 2 Producción total del biogás Fuente: Elaboración Propia

Se observa que el biodigestor número 2 presentó la mayor cantidad de biogás, seguido del 4 y del 3. Sin embargo, esto no indica que sean las mejores opciones para producir metano hasta conocer su porcentaje, ya que no se indica la calidad del biogás y si este puede ser considerado como un combustible.

HERNÁNDEZ-GÓMEZ, Víctor Hugo, OLVERA-GARCÍA, Omar y OAXACA-BELTRÁN, Marlene. Obtención de metano por medio de la combinación de excretas de vacas y conejos. Revista de Energía Química y Física. 2019. 
La temperatura juega un papel crucial en la taza de producción del biogás, ya que determina la velocidad con la que se produce y proliferan las bacterias que participan en el proceso. La figura 3 indica el rango de temperatura a la cual se encontraron los biodigestores, teniendo como valor mínimo 17.9 $\mathrm{C}$ y una temperatura máxima de $26.5 \mathrm{C}$, esto indica que las mezclas en los biodigestores se encontraron entre el rango psicrofilico y mesofilico.

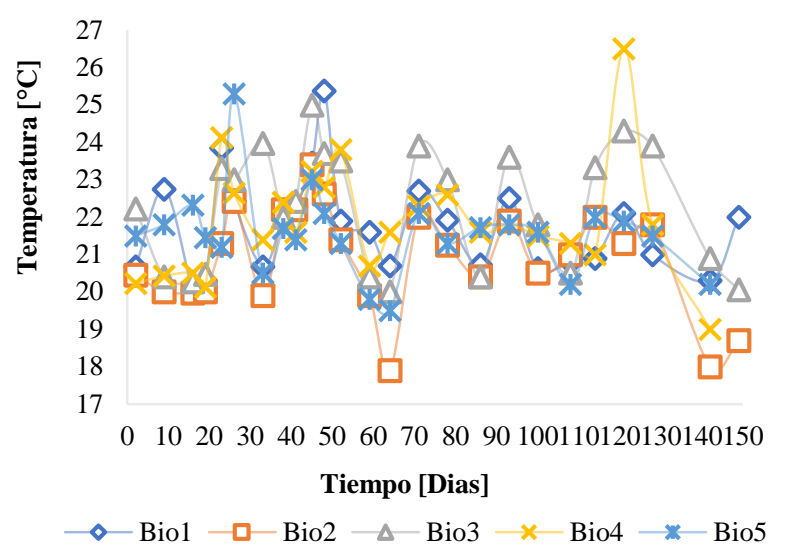

Figura 3 Temperaturas alcanzadas en el interior de los biodigestores

Fuente: Elaboración Propia

En la figura 4 se presenta el comportamiento del $\mathrm{pH}$ durante la prueba. Se observa que no hubo mucha variación, es decir al medir el $\mathrm{pH}$ este daba como valor 8 , el cual se encontraba dentro del rango aceptable, ya que, si el valor era superior a 8, la formación de amoniaco puede incrementarse notablemente, inhibiendo la actividad microbiana.

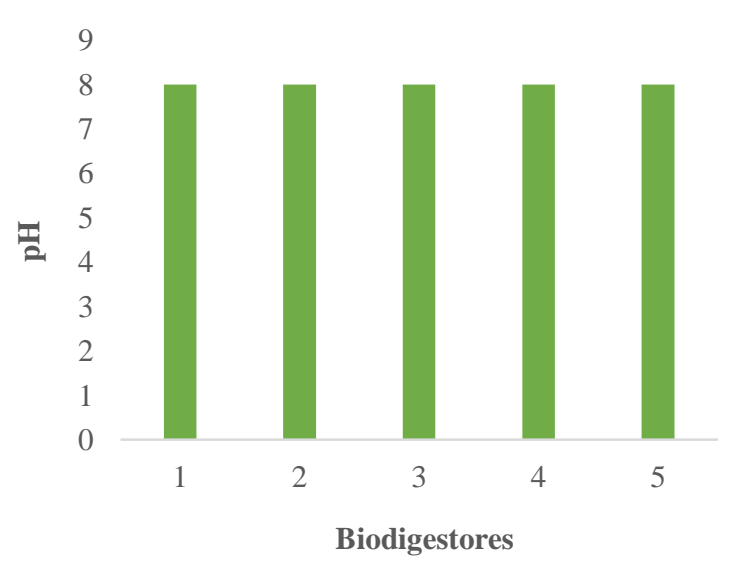

Figura 4 Valor del pH alcanzado en el interior del biodigestor

Fuente: Elaboración Propia

En la figura 5 se muestra la producción de metano $\left(\mathrm{CH}_{4}\right)$ de todos los biodigestores.

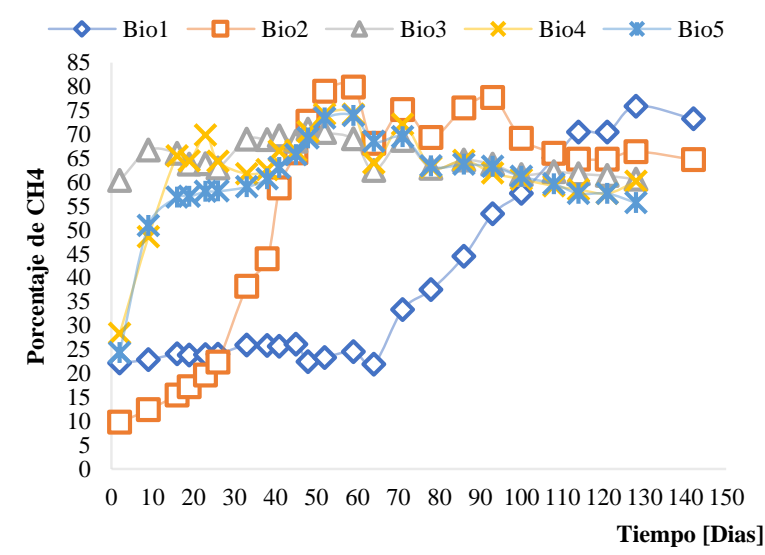

Figura 5 Porcentaje de metano obtenido en la prueba. Fuente: Elaboración Propia

Se observa que cada biodigestor reacciono de manera diferente, alcanzando el valor máximo en diferentes días. El bio1 presento $75.89 \%$ en el día 142 , el bio 2 presento $79.84 \%$ en el día 64 , el bio3 presento $71.04 \%$ en el día 52, el bio4 presento $74.23 \%$ en el día 59, el bio5 presentó $73.94 \%$ en el día 64, consecutivamente a estos días, el porcentaje de metano descendía, con excepción del bio4 el cual por una semana mantuvo su valor.

En la figura 6 se presentan los litros de metano producidos en cada semana y en la figura 7 se muestra la producción total de metano, deduciendo que, al mezclar las dos excretas, en diferentes porcentajes, resulta más eficiente, que cuando esta se encuentra sola.

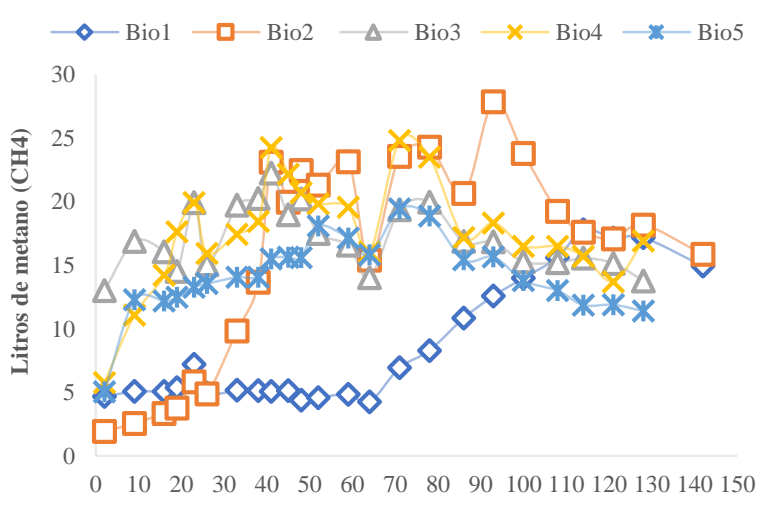

Tiempo [Dias]

Figura 6 Litros de metano $\left(\mathrm{CH}_{4}\right)$

Fuente: Elaboración Propia 


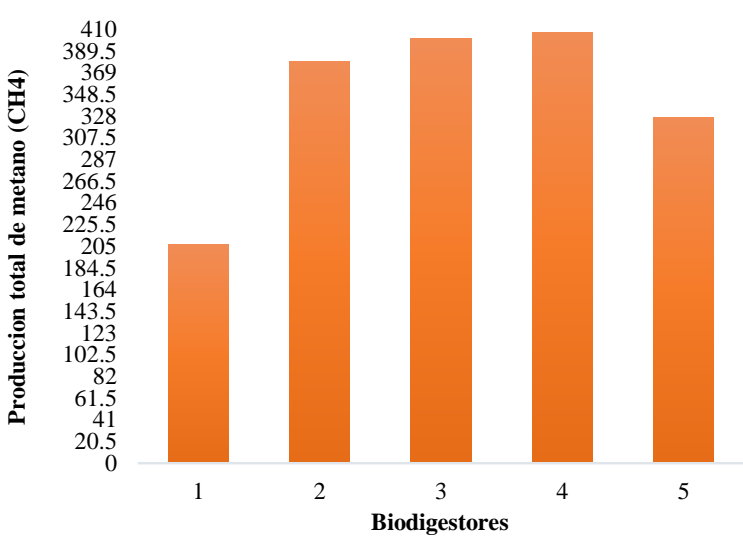

Figura 7 Producción total de metano Fuente: Elaboración Propia

Se observa que cada biodigestor respondió de diferente forma, algunos de los biodigestores alcanzaron el máximo valor en poco tiempo y después de esto el valor del metano descendía, otros alcanzaron su valor máximo en un tiempo más prolongado, ninguno de los biodigestores presento una estabilidad.

Comparando ambas figuras, entre dichas graficas existe una variación, observando que al principio de la prueba los biodigestores no presentaban altos valores de metano, pero si altos porcentajes de dióxido de carbono $\left(\mathrm{CO}_{2}\right)$, el cual no era de interés, después de ciertas semanas, la producción de metano supero al dióxido de carbono, creando un biogás con mayor calidad.

\section{Agradecimiento}

Agradecemos a la Universidad Nacional Autónoma de México, por el apoyo recibido para la realización del proyecto de investigación, a través del programa UNAM-DGAPA-PAPIMEPE101218.

\section{Conclusiones}

Con los resultados obtenidos se puede concluir que el biodigestor que presenta mayor generación de biogás con porcentaje de metano alto, es el biodigestor 4, alcanzando su máximo valor en el día 78. Su composición fue de $10 \%$ de excretas de vaca, $40 \%$ de excretas de conejo y $50 \%$ de agua.

Se observó que al combinar las excretas y realizando mezclas con diferentes porcentajes, se obtiene un biogás con mayor calidad, además que presentaban cierta estabilidad, comparándolo con los biodigestores que se hacían funcionar con un solo tipo de excreta.
El potencial de generación de gas puede considerarse para suministro a los laboratorios de la FESC.

\section{Referencias}

[1] Lopez, S; Calderón, S (2014): UF0285: Tratamientos de residuos urbanos municipales: España, Elearning S, L.

[2] Deublein, Dieter; Steinhauser, Angelika (2008): Biogas from waste and renewable resources: Alemania, Weinheim

[3] Hernández Gómez Víctor Hugo, Olvera García Omar, Espinal Arellano Juan Carlos y Morillón Gálvez David. Potencial de generación de biogás de un rancho ganadero en la comunidad de San Bartolo Cuautlalpan. Revista de Sistemas Experimentales 2016, 3-8: 36-52. ISSN: 2410-3950. Bolivia.

[4] Hernández Gómez Víctor, Olvera García Omar, Guzmán Tinajero Pedro y Morillón Gálvez David. Dimensionamiento de dos biodigestores para la producción de metano en la FES Cuautitlán, empleando la materia fecal de conejos y vacas. Revista del Desarrollo Tecnológico 2017, 1-3: 44-53. España.

[5] Hernández Gómez Víctor, Olvera García Omar, Gonzalez Aguirre Jesus y Gonzalez Maya Luis. Estudio del potencial de generación de metano empleando diferentes materias orgánicas de desecho. Ciencias de la Ingeniería y Tecnología, Handbooks T-VIII, 62-73. ISBN: 978-607-8534-73-9. Handbook editado por ECORFAN-México, S.C. (2018).

Manual granja integral autosuficiente/ hogares juveniles campesinos. Editorial San pablo.

Producción Semi-intensiva de cerdos y usos de desechos para generar energía. Miguel Chinchilla, Horacio Chi y Wilfrido Carrillo. Editorial MAG PRIAG. 